\title{
Schwere disseminierte Infektion durch Mycobacterium bovis BCG als Folge einer intravesikalen BCG-Therapie: Diagnostische Herausforderungen und therapeutische Optionen
}

\author{
Severe Disseminated Mycobacterium bovis BCG Infection as a Consequence of Intravesical BCG Therapy: \\ Diagnostic Challenges and Therapeutic Options
}

Autoren

Institut
P. Stais, K. Kambartel, A. Yildirim, T. Voshaar

Medizinische Klinik III, Krankenhaus Bethanien, Moers eingereicht $\quad 16.12 .2013$ akzeptiert nach Revision 18.3.2014

Bibliografie

Dol http://dx.doi.org/ 10.1055/s-0034-1365487 Online-Publikation: 13.5.2014 Pneumologie 2014; 68: 488-491 (c) Georg Thieme Verlag KC Stuttgart · New York ISSN 0934-8387

Korrespondenzadresse Dr. Patrick Stais Medizinische Klinik III Krankenhaus Bethanien Bethanienstr. 21 47441 Moers Patrick.Stais@bethanienmoers. de

\section{Zusammenfassung \\ $\nabla$}

Wir präsentieren den Fall eines 65-jährigen Patienten, der an einem oberflächlichen Harnblasenkarzinom leidet und infolge einer intravesikalen BCG-Therapie eine kritische systemische inflammatorische Reaktion aufgrund einer Disseminierung von Mycobacterium bovis BCG in Leber und Lunge erlitt. Neben der antituberkulotischen Therapie unter Beachtung einer Primärresistenz des M. bovis BCG gegenüber PZA diskutieren wir, basierend auf publizierten Fallberichten, die Möglichkeiten einer immunmodulierenden Therapie mit Steroiden [1].

\section{Hintergrund}

Ein 65-jähriger Patient, der seit Januar 2012 an einem oberflächlichen Harnblasenkarzinom leidet, unterzog sich im Juni 2012 einer adjuvanten intravesikalen BCG-Therapie. In der Folge entwickelte er eine systemische Reaktion im Sinne einer disseminierten pulmonalen Infektion mit Mycobacterium bovis BCG.

Die Diagnostik stellt den behandelnden Arzt vor eine Herausforderung, da selten ein Keimnachweis gelingt. Eine kürzlich publizierte Übersichtsarbeit von 15 Patienten, die an einer vergleichbaren systemischen Disseminierung von M. bovis BCG erkrankten, zeigt, dass eine PCR-basierte Erregerdiagnostik aus Vollblut, transbronchialer Biopsie oder Blasenbiopsie lediglich in $20 \%$ der Fälle einen positiven Befund hervorbringt. Auch eine transbronchiale Biopsie führt zu keinem wegweisenden Befund. Zwar findet man in 67\% der Fälle das histologische Bild nicht verkäsender Granulome; dieser Befund ist jedoch nicht als pathognomonisch für eine Infektion mit Mycobacterium BCG anzusehen. Lediglich in 1 von 15 Fällen erbrachte man den Nachweis von verkäsenden Granulomen [1].

\section{Abstract \\ $\nabla$}

We present the case of a 65 -year-old patient with superficial carcinoma of the bladder who developed systemic inflammatory reaction as a result of a severe disseminated infection with $M$. bovis BCG after intravesical instillation of BCG. Besides the recommended antituberculosis therapy, considering the resistance of M. bovis to PZA, we discuss here the option of using steroids in the therapeutic management of this patient.

Die Deutsche Gesellschaft für Pneumologie empfiehlt eine initiale Dreifachtherapie mit Isoniazid, Rifampicin und Ethambutol. In der Literatur wird, basierend auf einzelnen Kasuistiken, eine Steroidtherapie als supportive therapeutische Option zusätzlich erwähnt [2]. In unserer Kasuistik wollen wir insbesondere die diagnostischen Verfahren sowie auch die Therapieoptionen diskutieren.

\section{Fallbeschreibung}

V

\section{Anamnese}

Ein 65-jähriger Patient, der seit Januar 2012 an einem oberflächlichen Harnblasenkarzinom leidet (pTis Nx Mx G3), wurde uns im Juli 2012 aus einer benachbarten urologischen Abteilung zugewiesen.

Im Rahmen der Grunderkrankung hatte man wiederholte BCG-Instillationen vorgenommen, die bislang komplikationslos vertragen wurden. Aktuell aufnahmebegründend in die Urologie waren hohes Fieber, Dyspnoe und eine gravierende Beeinträchtigung des Befindens wenige Stunden nach ambulanter adjuvanter BCG-Instillation in die Blase, perakut einsetzend. 


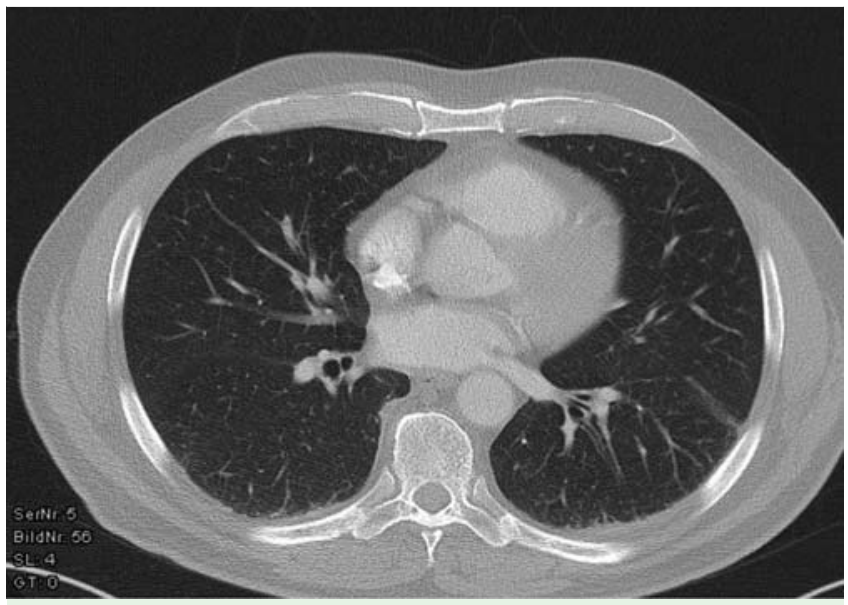

Abb. 1 Initiale CT-Aufnahme an Tag 6 nach stationärer Aufnahme.

Bei dem ansonsten gesunden Patienten ergab die Eigenanamnese einen Zustand nach Lungenabszess im Januar des Jahres 2011, die Medikamentenanamnese war leer, der Patient war Nichtraucher. Er ging einer Bürotätigkeit als Versicherungskaufmann nach. Unter der Annahme einer Harnwegsinfektion wurde zunächst kalkuliert antibiotisch mit Ciprofloxacin therapiert.

Aufgrund fehlenden Anschlagens $72 \mathrm{~h}$ nach Gabe der primären Antibiose ging man dann von einer Nebenwirkung der BCG-Instillation aus und therapierte über drei Tage mit der klassischen antituberkulotischen Vierfachtherapie (Isoniazid (INH), Rifampicin (Rifa), Ethambutol (EMB), Pyrazinamid (PZA)). Auch unter dieser Therapie litt der Patient weiter an Fieber, Fatigue, starkem Nachtschweiß und Belastungsluftnot.

Eine Computertomografie der Lunge an Tag 6 nach Aufnahme führte zu der Diagnose „interstitielle Pneumonie“ ( $\bullet$ Abb.1). Zunächst führten diese Befunde zu keiner Änderung der Therapie, weitere drei Tage später (Tag 9 nach Aufnahme) aber wurde die antimikrobielle Therapie aufgrund fehlenden Ansprechens bei persistierenden Beschwerden und weiterer Reduktion des Allgemeinbefindens empirisch auf Meropenem umgestellt.

Da auch dieser therapeutische Ansatz versagte und die Symptome persistierten, verlegte man den Patienten auf seinen Wunsch hin in unser Lungenzentrum.

\section{Körperlicher Untersuchungsbefund}

Wir sahen initial einen 65-jährigen Patienten in mittelgradig reduziertem Allgemeinzustand, Größe $188 \mathrm{~cm}$, Gewicht $96 \mathrm{~kg}$, Atemfrequenz 18/min. Unter Raumluft zeigte sich eine 95\%-ige Sauerstoffsättigung. Der Patient war nass geschwitzt, hypotherm (35,8 Grad C) und zittrig. Zyanotische Zeichen ließen sich nicht erkennen. Auskultatorisch fiel ein vesikuläres Atemgeräusch ohne Nebengeräusche auf. Die subjektiv führenden Symptome des Patienten waren Fieber, Fatigue und Belastungsluftnot.
Lungenfunktionsprüfung und kapilläre Blutgasanalyse unter Raumluft

\begin{tabular}{|c|c|c|}
\hline pO2 & $74 \mathrm{mmHg}$ & \\
\hline pCO2 & $32 \mathrm{mmHg}$ & \\
\hline pH & 7,46 & \\
\hline $\mathrm{SO} 2$ & $95 \%$ & \\
\hline TLC & 8,441 & $106 \%$ SW \\
\hline VC & 4,411 & $87 \%$ SW \\
\hline FEV1 & 3,21 & $86 \%$ SW \\
\hline FEV1\%VCin & $73 \%$ & $97 \%$ SW \\
\hline Rtot & $0,4 \mathrm{kPA}^{*} \mathrm{~s} / \mathrm{l}$ & $125 \%$ SW \\
\hline RV\%TLC & $48 \%$ & $123 \%$ SW \\
\hline DLCO & $7,99 \mathrm{mmol} / \mathrm{min} / \mathrm{kPa}$ & $76 \% S W$ \\
\hline $\mathrm{KCO}$ & $1,09 \mathrm{mmol} / \mathrm{min} / \mathrm{kPa} / \mathrm{l}$ & $82 \%$ SW \\
\hline
\end{tabular}

\section{Röntgen-Thoraxaufnahme in 2 Ebenen}

Diffuse feinnoduläre Zeichnungsvermehrung beider Lungen mit Betonung der Mittel- und Unterfelder. Mittelständiges und normal breites Mediastinum. Gefäßartig konfigurierte Hili. Herzgröße im Normbereich. Keine vitientypische Herzkonfiguration. Keine Pleuraergüsse. Überlagernder Weichteilschatten in den Unterfeldern ( $\bullet$ Abb. 2).

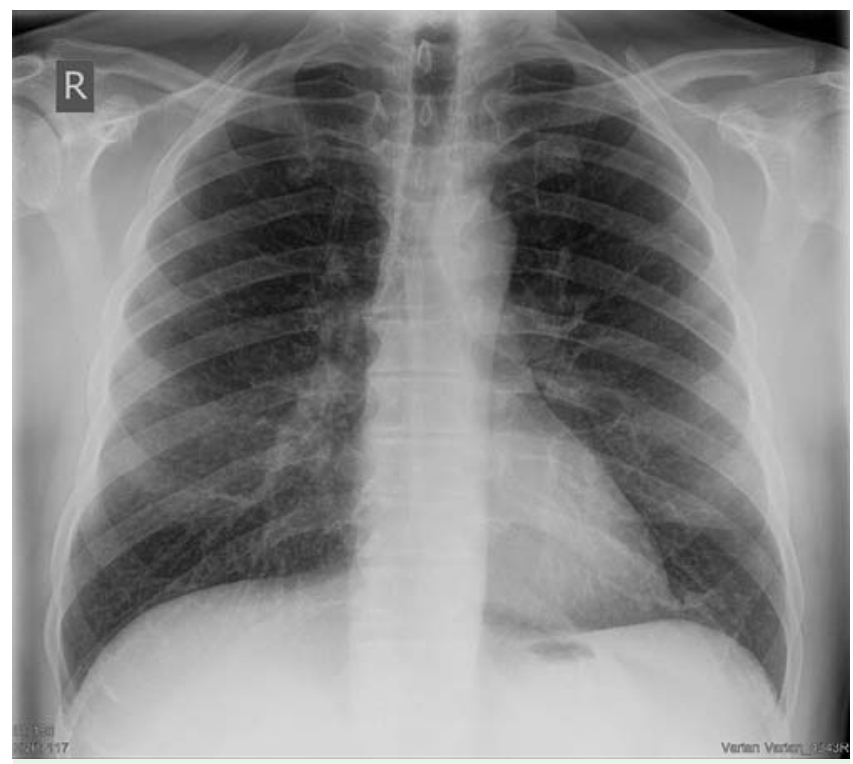

Abb.2 Auf der Röntgen-Thoraxaufnahme am Tag der Übernahme dominiert eine feinnoduläre Zeichnungsvermehrung.

\section{CT Thorax und Oberbauch mit Kontrastmittel (KM)} an Tag 21 nach Primäraufnahme

Hauptbefundlich finden sich im Lungenfenster multiple winzige Knötchen, die in der gesamten Lunge disseminiert zu sehen sind. Diese wurden sowohl im intra- als auch im interlobären Interstitium identifiziert und zeigen eine leichte Häufung in den basalen Lungenabschnitten beider Lungen. Zusammenfassend zeigt sich das Bild einer miliaren Aussaat.

Oben beschriebene Befunde sind auf der vorliegenden externen Voraufnahme in dieser Deutlichkeit nicht vorhanden gewesen. Nebenbefundlich zeigt sich eine größere Konsolidierungszone im rechten Lungenoberlappen, bei der es sich um ein narbiges Residuum der anamnestisch bekannten abszedierenden Pneumonie handeln dürfte ( $\bullet$ Abb.3). 


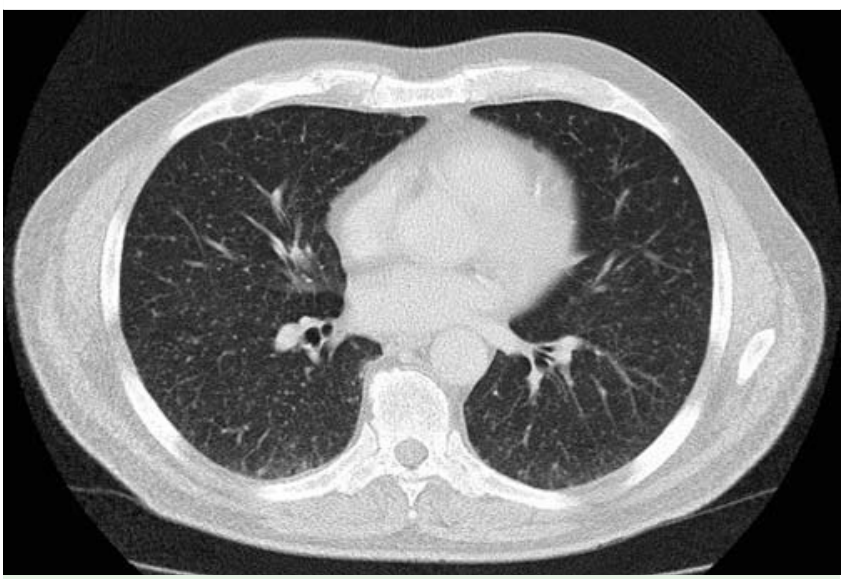

Abb. 3 Die Computertomografie an Tag 21 zeigt ein disseminiertes feinnoduläres hirseartiges Muster.

\section{Bronchoskopie}

Alle Ostien zeigen sich hier frei einsehbar. Keine akuten Entzündungszeichen. Keine Schleimhautgranulome. Kein Sekret. Keine Tumorzeichen. Da es sich um eine intrapulmonal disseminierte Erkrankung mit Befall sämtlicher Lungenlappen handelt, werden alle 5 Lappen mit jeweils $1 \mathrm{ml} \mathrm{NaCl}$ 0,9\% zur bakteriologischen und zytologischen Untersuchung angespült. Im Anschluss erfolgte eine BAL im Mittellappen.

\section{BAL}

Normale Gesamtzellzahl mit sehr guter Zellvitalität. Differenzialzytologisch starke lymphozytäre Alveolitis (50\%) mit normwertigem CD4/CD8-Quotienten (2,7) mit normwertiger Anzahl von NK-Zellen (1\%) sowie vermehrter Expression von T-Zellaktivierungsmarkern (IL-2-Rezeptor positiv 8\%), Einschlüsse exogenen Pigments in die Alveolarmakrophagen. Keine Bakterien. Keine Pilze. Keine suspekten Zellen oder Zellkomplexe.

\section{Tuberkulin-Hauttest nach Mendel/Mantoux}

Deutliche Induration nach 72 Std. mit einem Diameter von ca. $1,5 \mathrm{~cm}$.

Interferon-Gamma-Release-Essay/Quantiferon-Gold-Test Interferon-Gamma negativ-Kontrolle 2,06 IU/ml. IFN-GammaTB-Antigen minus negativer Kontrolle:-0,03 IU/ml (Referenzbereich $<0,35$ ). Interferon-Gamma positiv, Kontrolle 15,5 IU/ml. Testausfall somit negativ.

\section{Zelluläre und humorale Immunologie}

CD4/CD8-Quotient der Blutlymphozyten in der Norm (2,5; Referenzbereich 2,9).

HIV-Test negativ. Immunglobuline quantitativ normwertig: IgA $335 \mathrm{mg} / \mathrm{dl}$; IgM $423 \mathrm{mg} / \mathrm{dl} ;$ IgG $955 \mathrm{mg} / \mathrm{dl}$.

\section{Mikrobiologische Verfahren}

Mit $1 \mathrm{ml}$ 0,9\%iger Kochsalzlösung verdünntes Bronchialsekret aus sämtlichen Lungenlappen:

Untersucht wurde mikroskopisch auf säurefeste Stäbchen, eine Kultur auf Mykobakterien angelegt und eine PCR auf Mycobacterium-tuberculosis-Komplex veranlasst. Sämtliche Befunde waren sowohl primär als auch nach 8 Wochen negativ.

\section{Material Urin (10 ml, Vorderstrahl)}

Kultur auf Mykobakterien, PCR auf Mycobacterium-tuberculosisKomplex: Tb PCR schwach positiv, Kultur nach 8 Wochen negativ.

\section{Blutkulturen 2 Paar}

Kultur auf Mycobacterium nach 8 Wochen negativ. Mikroskopie auf säurefeste Stäbchen negativ.

\section{Laborchemische Routineparameter}

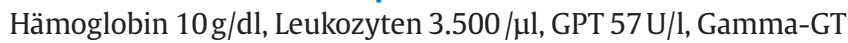
$170 \mathrm{U} / \mathrm{l}$, CRP 1,4 mg/dl. Procalcitonin 0,07 pg/ml, Hepatitisserologie negativ.

\section{Verlauf \\ $\nabla$}

Wir formulierten bei dem hier beschriebenen Fall die Arbeitsdiagnose einer disseminierten pulmonalen Infektion mit Mycobacterium bovis BCG. Hierfür sprach neben der Radiomorphologie insbesondere die Anamnese mit einer Beschwerdesymptomatik, die perakut nach der letzten Instillation der intravesikalen BCG-Instillation auftrat.

Nach entsprechender Diagnostik setzten wir die im zuweisenden Krankenhaus begonnene und bei fehlendem Ansprechen nach insgesamt 6 Tagen unterbrochene antimykobakterielle Therapie als 3-fach Therapie fort. Pyrazinamid wurde aufgrund der Primärresistenz von M. bovis nicht erneut gegeben. Auch unter dieser Therapie kam es zu keiner adäquaten Entfieberung und Regredienz des Nachtschweißes, sodass wir uns nach 5 Tagen antimykobakterieller Therapie zu einer zusätzlichen Steroidtherapie mit zunächst $100 \mathrm{mg}$ Prednisolonäquivalent/Tag als Stoßtherapie über einen Zeitraum von drei Tagen entschlossen, um einer überschießenden, hyperergen Immunreaktion entgegenzuwirken. Hierunter kam es zu einer anhaltenden Entfieberung, Besserung des Allgemeinbefindens, deutlicher Rückläufigkeit des Nachtschweißes und zu einer besseren Belastbarkeit, sodass wir die Glukokortikoide oral in einer Dosis von $30 \mathrm{mg}$ Prednisolonäquivalent per diem weiter verordneten. Die Therapie war primär auf 9 Monate Behandlungsdauer ausgelegt.

In subjektiv deutlich gebessertem Allgemeinzustand konnten wir den Patienten dann 14 Tage später in die ambulante hausärztliche und von uns fachärztlich begleitete Weiterbetreuung entlassen.

\section{Diskussion \\ $\nabla$}

Die intravesikale BCG-Instillation hat sich in der adjuvanten Therapie des nicht-muskelinvasiven Blasenkarzinoms als Standardtherapie seit gut 35 Jahren etabliert [3].

Der Bazillus Calmette-Guérin ist ein attenuierter Erreger, welcher Anfang des 20. Jahrhunderts aus Mycobacterium bovis gezüchtet wurde. Kommerziell verfügbare BCG-Lösungen enthalten zwischen $2 \times 10^{8}$ bis $3 \times 10^{9}$ lebensfähige Einheiten von BCG (Bacillus Calmette-Guérin)-Bakterien. Nach Instillation kommt es zu einer immunologischen Antwort mit Freisetzung von Interferon Gamma durch Induktion von TH1-Helferzellen [4].

In der Regel ist die Therapie gut verträglich, 95\% der Patienten zeigen nach der Therapie keine signifikanten Krankheitszeichen. Wenn Nebenwirkungen auftreten, sind dies üblicherweise Fieber oder lokale Reaktionen, die zu Dysurie oder Hämaturie führen können. In der überwiegenden Anzahl der Fälle lassen sich diese 
Symptome durch symptomatische Maßnahmen kontrollieren und bedürfen keinerlei spezifischer Therapie [5]. Extravesikale Nebenwirkungen oder Komplikationen wie eine granulomatöse Prostatitis (bis zu 40\%, oft asymptomatisch), eine EpididymitoOrchitis $(<1 \%)$, eine Hepatitis $(<1 \%)$, eine Pneumonitis $(<1 \%)$ oder gar systemische Verläufe wie eine disseminierte BCG-Sepsis $(0,4 \%)$ hingegen sind selten beschrieben worden und bedürfen einer raschen antimykobakteriellen Therapie [6]. Man nimmt an, dass ca. einer von 15000 Patienten eine solche schwere systemische Verlaufsform erleidet [7].

Legt man eine Literaturrecherche in PubMED mit den Schlagwörtern BCG und Pneumonitis zugrunde, so findet man bis August 201335 Fälle, in denen eine „miliare Pneumonitis“ beschrieben wird. Beispielhaft sei hier nochmals die Übersichtsarbeit von Caramori et al. erwähnt [1].

Die Diagnose einer BCG-itis stützt sich primär auf die Anamnese und die Bildgebung. Das Erkennen dieses seltenen Krankheitsbildes setzt daher die entsprechenden Kenntnisse voraus. Eine weitere diagnostische Absicherung kann durchaus eine Herausforderung darstellen, da ein Keimnachweis aus dem Sputum oder der Bronchiallavage wegen der primär hämatogenen Streuung nicht gelingt. Auch in den oben genannten Publikationen gelang ein Keimnachweis nur in $20 \%$ der Fälle, und zwar nur via PCR [1].

Bezüglich einer histologischen Sicherung gibt es derzeit keinerlei offiziellen Empfehlungen, es scheint uns aber auch nicht hilfreich, diese einzufordern, da das Nutzen-Risiko-Verhältnis sicher ungünstig ist und der diagnostische Zugewinn bei einer vom Pathologen beschriebenen hyperergen, granulomatösen Reaktion der Lunge gering ist. In lediglich 1 von 15 Fällen konnten verkäsende Granulome nachgewiesen werden [1].

Einen endobronchialen Bakteriennachweis darf man primär nicht erwarten. Die miliare Aussaat (Milien= „Hirsekörner“) ist gekennzeichnet durch diffuse Knötchen in der Lunge, die infolge der hämatogenen Bakterienaussaat typischerweise keinen Anschluss an das Bronchialsystem haben. Erst bei einer Desintegration eines solchen Granuloms, könnte ein Bakteriennachweis endobronchial gelingen.

Eine Herausforderung an den behandelnden Arzt kann die Therapiestrategie sein.

Das DZK und die DGP nehmen in ihren Empfehlungen zur Therapie, Chemoprävention und Chemoprophylaxe sowohl zur „BCGitis“ als Lokalreaktion sowie zur disseminierten BCG-Infektion Stellung [8]. Hier wird eine Dreifachtherapie aus Isoniazid, Rifampicin und Ethambutol über mindestens 6 Monate empfohlen, wobei eine Prolongation auf 9 Monate Therapiedauer aus Gründen der Primärresistenz des Mycobacterium bovis BCG auf Pyrazinamid empfohlen wird.

In der internationalen Literatur findet man Einzelfallberichte, bei denen zusätzlich mit systemischen Steroiden behandelt worden ist.

Die Rationale hierfür geht von der Hypothese aus, dass die akute klinische Symptomatik einer systemischen „BCG-itis“ in erster Linie durch eine hypererge Reaktion infolge der massiven hämatogenen Aussaat der Bakterien bestimmt wird. Wir entschlossen uns, vor dem Einsatz von Glukokortikoiden zunächst mit der antituberkulotischen Therapie zu beginnen. Erst als diese innerhalb von 5 Tagen zu keiner symptomatischen Besserung führte und die Beeinträchtigung des Patienten weiter erheblich war, begannen wir mit einer Steroidtherapie. Wir halten dieses Vorgehen auch im Nachhinein für eine adäquate Entscheidung, da die sofort gestartete antimykobakterielle Therapie natürlich entscheidend ist und einen gewissen Sicherheitskorridor schafft, bevor gegebenenfalls auch eine Immunsuppression begonnen wird. Kurze Zeit nach dem hier dargestellten Fall haben wir einen weiteren Patienten mit einer „BCG-itis“ und fast identischem Verlauf behandelt. Allerdings haben wir bei diesem Patienten mit ebenfalls schwerer Beeinträchtigung des Allgemeinbefindens und akut einsetzender Lungenfunktionseinschränkung die immunsuppressive Therapie direkt mit der antimykobakteriellen Behandlung begonnen. Unter diesem Vorgehen fiel die Zeit der unspezifischen Beschwerden wie Fieber, Nachtschweiß, Schwäche etc. im Vergleich zum hier ausführlich dargestellten Kasus deutlich kürzer aus.

Nach unserer Auffassung sollte der Beginn einer Glukokortikoidtherapie parallel zur antituberkulotischen Behandlung bei schweren Allgemeinsymptomen und bedeutsamer akuter Einschränkung, insbesondere des Gasaustausches, nicht verzögert werden. Dieses Vorgehen erscheint uns im Übrigen auch hinsichtlich der primär infektiösen Genese vertretbar und kann in Einzelfällen möglicherweise sogar lebensrettend sein. Dies gilt insbesondere in Hinblick auf die in der Literatur beschriebenen schweren Verlaufsformen mit ARDS [9].

Kontrollierte Studien zum besseren Verständnis der seltenen Erkrankung wären wünschenswert, um therapeutische Empfehlungen auf wissenschaftlich fundierte Evidenz zu stützen.

\section{Interessenkonflikt}

$\nabla$

Die Autoren geben an, dass kein Interessenkonflikt besteht.

\section{Literatur}

1 Caramori G, Artioli D, Ferrara $G$ et al. Severe pneumonia after intravesical BCG instillation in a patient with invasive bladder cancer: case report and literature review. Monaldi Arch Chest Dis 2013; 79: 44 - 48

2 Uetsuki H, Hirama H, Matsuoka Y et al. A case with hepatitis and interstitial pneumonitis caused by intravesical bacillus Calmette-Guérin (BCG) instillation. Nihon Hinyokika Gakkai Zasshi 2011; 102: 691 - 695

3 Sylvester RJ. Bacillus Calmette-Guérin treatment of non-muscle invasive bladder cancer. Int J Urol 2011; 18: 113 - 120 [Epub 2010 Nov 22]

4 Askeland EJ, Newton MR, O'Donnell MA et al. Bladder Cancer Immunotherapy: BCG and Beyond. Adv Urol 2012: 181-987 [Epub 2012 Jun 20]

5 Rodríguez F, Palou J, Martínez $R$ et al. Practical guideline for the management of adverse events associated with BCG installations. Arch Esp Urol 2008; 61: 591 - 596

6 Koya MP, Simon MA, Soloway MS. Complications of intravesical therapy for urothelial cancer of the bladder. J Urol 2006; 175: 2004-2010

7 Lamm DL. Complications of bacillus Calmette-Guérin immunotherapy. Urol Clin North Am 1992; 19: 565-572

8 Schaberg T, Bauer T, Castell S et al. Recommendations for therapy, chemoprevention and chemoprophylaxis of tuberculosis in adults and children. German Central Committee against Tuberculosis (DZK), German Respiratory Society (DGP). Pneumologie 2012; 66: 133-171

9 Rival G, Garot D, Mercier E et al. Acute respiratory failure and septic shock induced by Mycobacterium bovis. A rare side effect of intravesical BCG therapy. Presse Med 2006; 35: 980-982 